\title{
PRÉ-SELEÇÃO DE ESTIRPES DE RHIZOBIUM SP. PARA AMENDOIM (1)
}

\author{
ANTONIO ROBERTO GIARDINI (2) e ELI SIDNEY LOPES (2), Seção de Mierobiologia do \\ Solo, Instituto Agromómico, e ANDRE MARTIN LOUIS NEPTUNE (3), Escola Superior de \\ Agricultura "Luiz de Queiroz", Universidade de São Paulo.
}

\begin{abstract}
RESUMO
Um ensaio foi conduzido em casa de vegetação, com solução nutritiva isenta de $\mathrm{N}$, com o objetivc de selecionar estirpes de Rhizobium eficientes fixadoras de $N_{\text {, }}$, quando associadas com amendoim (Arachis hypogaea L.) cultivar Tatu. Foram testadas 35 estirpes de Rhizobium sp., isoladas de quinze diferentes espécies de ieguminosas tropicais, e incluído um tratamento de inoculação com solo previamente cultivado com amendoim. Das 35 estirpes testadas, doze formaram nódulos e, entre essas, sete foram eficientes fixadoras de nitrogênio. Das doze estirpes que nodularam, sete foram isoladas de leguminosas da tribo Hedysareae (à qual pertence - gênero Arachis) e, destas, apenas quatro foram eficientes fixadoras de nitrogênio. o peso e o número de nódulos não se mostraram como critérios adequados para avaliação da eficiência.
\end{abstract}

\section{INTRODUCĀO}

$\mathrm{O}$ amendoim tem sido pouco estudado em nossas condiçōes, quanto ao aproveitamento de sua capacidade de fixar o $\mathrm{N}_{2}$ atmosférico. Por esse motivo, ainda se fazem necessárias as recomendaçōes para aplicação de adubo nitrogenado nessa cultura (13).

(1) Parte da tese de mestrado do primeiro autor na ESALQ. Apresentado na 9a Reuniāo Latino-Americana Sobre Rhizobium - RELAR - México. Trabalho realizado com o apoio financeiro do Projeto Amendoim - Convênio EMBRAPA/SAA. Recebido para publicação a 2 de marco de 1983.

(2) Bolsista do CNPq.

(3) Professor da ESALQ - USP. 
Para uma área de plantio com 211.000 hectares aproximadamente, considerando-se o amendoim da seca e das águas, no ano de 1980, calcula-se que foram aplicadas cerca de 1.000 toneladas de $\mathrm{N}$ nessa cultura $\left(\operatorname{LASCA}^{4}\right)$.

Em vista da substituição dessa oleaginosa por cutras de maior rentabilidade, como a soja, a área cultivada vem decrescendo sensivelmente nos últimos anos (12). Observações esporádicas revelam que o amendoim nodula abundantemente em condições de cultivo. LOPES et alii (9) verificaram nodulação espontânea, aparentemente eficiente, em 21 cultivares dessa leguminosa numa mesma área experimental. Em teste anterior para seleção de estirpes eficientes fixadoras de $\mathrm{N}_{2}$, foi observado que apenas três entre nove estirpes de Rhizobium sp. nodularam o amendoim (8).

No presente trabalho são apresentados resultados cie um teste de eficiệncia de fixação com 35 estirpes.

\section{MATERIAL E MÊTODOS}

O ensaio, conduzido em casa de vegetação, utilizando-se vasos de Leonard, com solução nutritiva isenta de nitrogênio, conforme a técnica recomendada por NORRIS (11), foi realizado com o cultivar Tatu, o mais plantado no Estado de São Paulo. Foram feitos 32 iratamentos de inoculação com as estirpes de Rhizobium sp. abaixo inơicadas, e três tratamentos adicionais: um de inoculação com solo previamente cultivado com amendoim e dois controles sem inoculação, sendo um sem $\epsilon$ outro com nitrogênio combinado.

Ieguminosa de origem

Aeschynomene paniculata Willd *

Aeschynomene sp. *

Arachis hypogaea L.*

Desmodium uncinatum (Jacq.) DC. *

Desmodium pabulare (Hoehne) Malme *

Macrotyloma africanus (Wilczeck) Verdc.

Lablab purpureus (L.) Sweet

Dolichus sp. n' SMS da estirpe

338
42
69 (= SA/RS ${ }^{\circ}$ 602), 124, 400
(= 411/MAR. Zimbabwe)
$2,3,193$
11
139 (= CB 756, Austrália)
311 (= IPEACs, Do 46)
110

(4) Eng?-Agro Dalmo H.C. Lasca - Assistente de Planejamento Agropecuário II da Coordenadoria de Assistência Técnica Integral (CATI) - Informação pessoal. 
Leguminosa de origem

Glycine wightii ( $R$. Grah,

Wight et Arn. Ver.)

Macroptilium atropurpureus (DC.) Urban

Phaseolus caracalla L.

Phaseolus erythroioma Mart. ex. Benth.

Phaseolus sp.

Stylosanthes guyanensis (Aubl.) Sw. *

Stylosanthes sp. * $n^{\circ}$ SMS da estirpe

12,137 ( $=\mathrm{CB} 4536,176$ ( $=\mathrm{D} 176$, Checoslováquia), 177 (= QA 422, Austrália), 213, 215, 221, $224,243,259$ e 280

$114,123,287,319,320$

118

6

19 e 289

312 (- IPEACs H-8)

55, 330 (= IPEACs S-5)

Essa relação inclui todas as estirpes isoladas de plantas da tribo Hedysareae disponíveis na coleção da Seção de Microbiologia do Solo (LOPES, 5), além de algumas de outras tribos testadas anteriormente em siratro e soja-perene (dados não publicados).

As sementes foram esterilizadas com solução de bicloreto de mercúrio 1:1.000, por três minutos e lavadas dez vezes con água esterilizada. Plantaram-se cinco sementes por vaso e, seis dias após, fez-se o desbaste, deixando-se duas plantas por vaso. A inoculação das culturas puras de Rhizobium foi feita um dia após o desbaste, com $2 \mathrm{ml} /$ vaso de caldo com bom crescimento das bactérias. Para o tratamento de inoculação com solo, utilizaram-se $10 \mathrm{ml}$ de uma suspensão de Latossolo Roxo a $10 \%$, em água esterilizada. Decorridos dez dias do plantio, os cotilédones de todas as plantas foram removidos para antecipar as deficiências de nitrogênio e, aos 14 dias, fez-se uma aplicação de nitrogênio no tratamento com adição daquele nutriente, usando-se $10 \mathrm{ml}$ de uma solução esterilizada de $\mathrm{NH}_{4} \mathrm{NO}_{3}$ a $1,42 \%$ por vaso. Outras aplicações foram feitas aos $20,28,36,42$ e 54 dias após o plantio, totalizando $298 \mathrm{mg}$ de nitrogênio por vaso.

O experimento foi instalado no mês de abril: durante os 45 dias finais de sua condução, registrou-se a temperatura, a $7 \mathrm{~cm}$ de profundidade, em dois vasos, utilizando-se um termógrafo Foxboro.

Na colheita aos 60 dias após o plantio, foram feitas determinações de peso da planta e de nódulos secos a $60^{\circ} \mathrm{C}$. Foi também feita análise de $\mathrm{N}$ na matéria seca, em auto-analisador Tecnicon II, segundo método descrito por BATAGLIA et alii (1).

Adotou-se o delineamento inteiramente casuaiizado com seis repetiçōes. Para as análises do número e peso dos nódulos, os valores foram transformados em $\sqrt{\mathrm{x}+1}$.

(*) Tribo Hedysareae. 


\section{RESULTADOS E DISCUSSÃO}

Pode-se verificar pela curva das médias horárias de temperatura que o experimento foi conduzido em condiçōes amenas (Figura 1). A máxima das médias, $28,7^{\circ} \mathrm{C}$, ocorreu às 14 horas. A temperatura esteve acima de $25^{\circ} \mathrm{C}$ das 11 às 19 horas, durante dez dias, entre o $18^{\circ}$ e o $32^{\circ}$ dia do plantio.

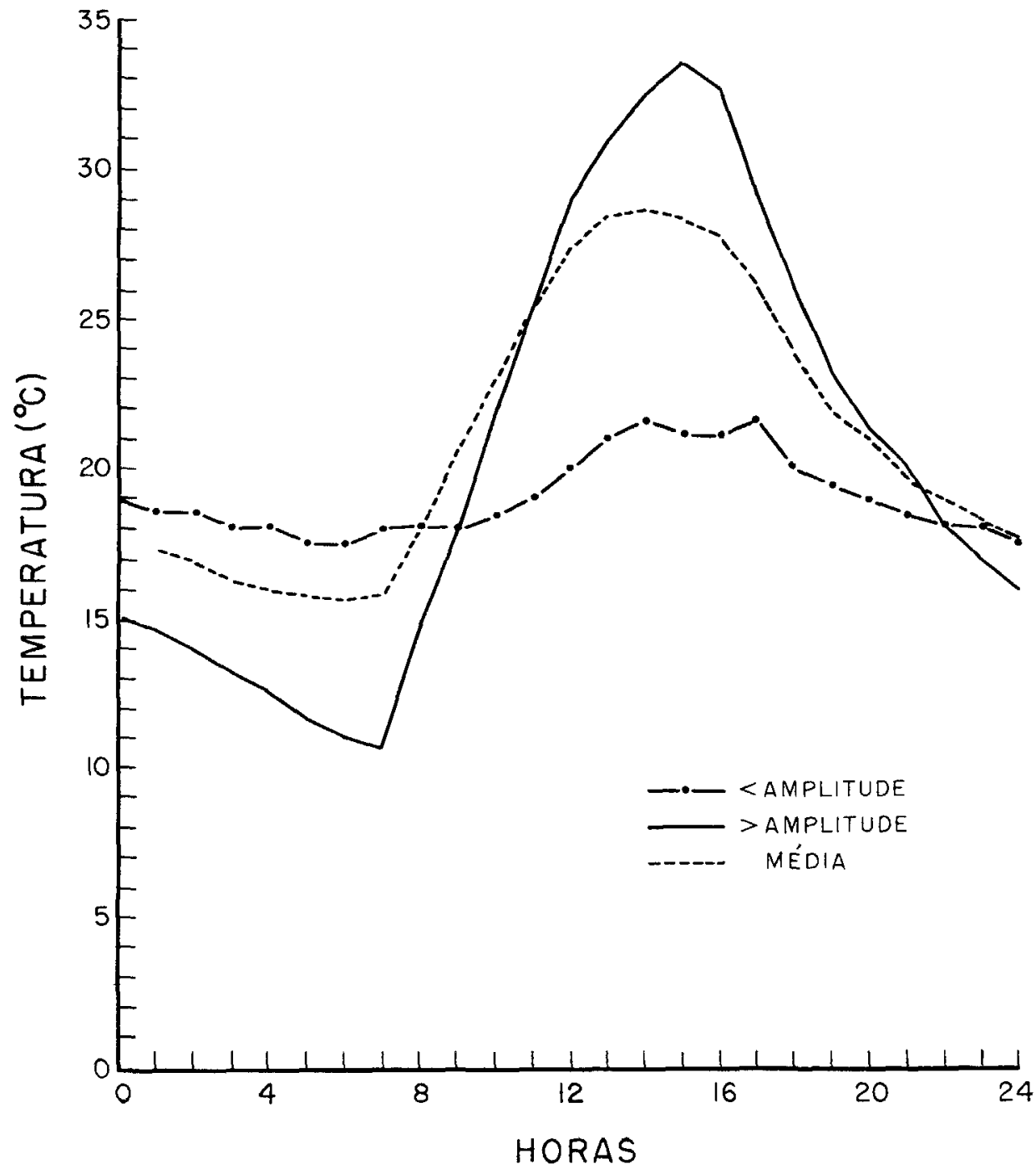

FIGURA 1. Temperaturas médias horárias para os quarenta e cinco dias finais do experimento e temperaturas dos dias de maior e menor amplitude de variação. 
Aos 28 dias após o plantio foram observadas pequenas manchas de cor marrom-escura, principalmente nas folhas mais velhas: de todas as plantas, e que persistiram até o final do experimento, mas cuja causa não foi possível identificar.

O início da fixação do nitrogênio, notado pela cor verde mais escura, em relação ao das plantas não inoculadas, foi observado entre 12 e 24 dias após o plantio, dependendo da estirpe. $O$ florescimento iniciou-se aos 31 dias.

No quadro 1, estão os dados de peso e número de ródulos, peso das plantas secas, nitrogênio total da parte aérea e quantidade de nitrogênio relativa ao controle que recebeu este nutriente en forma combinada.Os controles com e sem nitrogênio não nodularam, indicando que não houve problemas de contaminaçōes.

QUADRO 1. Número e peso seco de nódulos, peso seco e $\mathrm{N}$ total da parte aérea, e relação percentual entre $\mathrm{N}$ fixado e $\mathrm{N}$ do controle em duas plantas de amendoim

\begin{tabular}{|c|c|c|c|c|c|}
\hline \multirow[b]{2}{*}{$\begin{array}{l}\text { ESTIRPES } \\
\text { SMS }\end{array}$} & \multicolumn{3}{|c|}{$P A R T E A E E A$} & \multicolumn{2}{|c|}{ NODULAÇÃO } \\
\hline & $\begin{array}{l}\text { Matéria } \\
\text { seca }\end{array}$ & $\begin{array}{c}N \text { total } \\
\text { da parte } \\
\text { aérea }\end{array}$ & $\begin{array}{l}\text { Percentagem } \\
\text { relativa ao } \\
\text { controle }+ \text { iv }\end{array}$ & Número (1) & Peso (1) \\
\hline & g/vaso & $\mathrm{mg} / \mathrm{vaso}$ & & & \\
\hline 2 & $4,35 \mathrm{ab}$ & $164,8 \mathrm{ab}$ & 74 & $13,3 \mathrm{~b}$ & $10,7 \mathrm{~b}$ \\
\hline 42 & $4,47 \mathrm{ab}$ & $167,8 \mathrm{ab}$ & 75 & 17,3 a & $11,6 \mathrm{~b}$ \\
\hline 55 & $1,35 \mathrm{~d}$ & $29,4 \mathrm{~d}$ & 13 & $14,6 \mathrm{ab}$ & $9,1 \mathrm{~b}$ \\
\hline 123 & $1,58 \mathrm{~cd}$ & $32,3 \mathrm{~d}$ & 14 & $3,3 \mathrm{c}$ & $2,4 \mathrm{c}$ \\
\hline 124 & $1,45 \mathrm{~d}$ & $29,3 \mathrm{~d}$ & 13 & $12,9 \mathrm{~b}$ & $12,2 \mathrm{ab}$ \\
\hline 139 & $4,50 \mathrm{ab}$ & $186,1 \mathrm{ab}$ & 84 & $13,5 \mathrm{~b}$ & $11,7 \quad b$ \\
\hline 176 & $4,65 \mathrm{ab}$ & 198,6 a & 89 & $14,8 \mathrm{ab}$ & $11,0 \quad b$ \\
\hline 311 & $3,22 \mathrm{bc}$ & $125,2 b^{\prime}$ & 56 & $14,5 \mathrm{ab}$ & 14,4 a \\
\hline 312 & $4,62 \mathrm{ab}$ & $171,0 \mathrm{ab}$ & 77 & $15,8 \mathrm{ab}$ & $11,1 b$ \\
\hline 319 & 5,45 a & 225,9 a & 101 & $14,6 \mathrm{ab}$ & $13,0 \mathrm{ab}$ \\
\hline 330 & $1,23 \mathrm{~d}$ & $26,2 d$ & 12 & $6,2 \mathrm{c}$ & $4,6 \mathrm{c}$ \\
\hline 400 & 4,98 a & 212,5 a & 95 & $13,2 b$ & $11,1 \mathrm{~b}$ \\
\hline Solo & $1,88 \mathrm{~cd}$ & $57,7 \mathrm{~cd}$ & 26 & $5,9 \mathrm{c}$ & 9,1 b \\
\hline Controle & $1,18 \mathrm{~d}$ & $21,9 \mathrm{~d}$ & 10 & 0 & 0 \\
\hline Controle $+\mathrm{N}$ & 5,58 a & 222,9 a & 100 & 0 & 0 \\
\hline DMS & 1,67 & 68,5 & - & 3,62 & 2,68 \\
\hline $\mathrm{CV}$ & $24,59 \%$ & $27,0 \%$ & $\longrightarrow$ & $14,80 \%$ & $13,40 \%$ \\
\hline
\end{tabular}

(1) Dados transformados em $\sqrt{x+1}$. 
Das 35 estirpes testadas, somente doze nodularam. Destas, sete foram estatisticamente semelhantes ao controle que recebeu nitrogênio, quanto ao peso e nitrogênio total da parte aérea. A parte aérea das plantas do tratamento com nitrogênio continha em média $222,9 \mathrm{mg}$ de $\mathrm{N}$ /vaso e a dos inoculados com as melhores estirpes continha entre 164,8 (SMS-2) e 225,9mg de $\mathrm{N} /$ vaso (SMS-319). Os dados de teores de nitrogênio nas raízes nāo foram incluídos por não alterarem a interpretaçāo feita em base apenas no nitrogênio da parte aérea. $O$ total de nitrogênio na parte aérea das plantas do controle mais nitrogênio foi $257,5 \mathrm{mg}$ /vaso (34,5mg relativo às raízes). Isso indica que, até a época amostrada, $86 \%$ do nitrogênio adicionado havia sido assimilado pelas plantas daquele tratamento. As estirpes de menor eficiência foram as SMS-55, 123, 124, 311 e 330. As estirpes do solo também foram ineficientes.

Para um critério arbitrário, que considere como eficientes as estirpes que incorporem até $80 \%$ do total de nitrogênio do controle que recebeu esse nutriente, seriam consideradas eficientes apenas as estirpes SMS-319, 400, 176 e 139. Em ensaio anterior (8), as estirpes SMડ̄-139, 176 e 2 haviam-se mostrado eficientes em amendoim.

Os dados do quadro 1 revelam que os dados de peso de plantas e de nitrogênio separaram, de maneira geral, as mesmas estirpes. É interessante observar que o período que demorou para as plantas mostrarem indícios de fixação após a inoculação foi variável. A SMS-2 antecipou-se às demais, apresentando coloração verde mais intensa, aos doze dias da inoculação, enquanto as estirpes SMS-139, 176 e 311 apresentavam cor verde mais intensa somente aos 24 dias. Essa diferença de doze dias pode ser importante em condiçōes ce campo, visto que maior disponibilidade de nitrogênio nessa fase pode yromover maior desenvolvimento inicial da planta, com conseqüente maior capacidade de captação da energia necessária à fixação.

A SMS-176 havia se mostrado ineficiente em siratro e soja perene (trabalho não publicado) e não formara nódulos em galáxia (8); a SMS-139 fora ineficiente em soja-perene (dois ensaios näo publicados) e galáxia (8), e a SMS-2 fora eficiente em galáxia (8).

Os dados de nodulação (peso e número de nódulos secos) não se revelaram como critério adequado para separar as estirpes, como observado anteriormente para feijão, feijão-vagem, ervilha (não publicado) e soja (7). O número de nódulos das melhores estirpes variou entre 175 e 301 e o peso de nódulos entre 125,7 e $135,8 \mathrm{mg}$.

A maior quantidade de nódulos foi produzida pela estirpe SMS-42, que, entretanto, apresentou eficiência apenas razoável. A maior produção de massa nodular foi da estirpe SMS-311, sendo esta, contudo, pouco eficiente na fixação de $\mathrm{N}_{2}$.

Os nódulos formados pela população autóctone foram pelo menos duas vezes e meia maiores que os das estirpes das culturas puras, mas 
a nođulação foi inexpressiva. A SMS-55, de nodulação abundante e ineficiente, formou os nódulos de menor peso (em média $0,37 \mathrm{~g} /$ nódulo). A SMS 124, que havia apresentado, no cultivar Tatu, nódulos ineficientes de de grande tamanho (10), apresentou nódulos ineficientes, porém de tamanho normal (2 a $3 \mathrm{~mm}$ de diâmetro).

Somente quatro das sete estirpes isoladas de plantas da tribo Hedysareae que nodularam o cultivar Tatu (SMS-2, 42, 312 e 400) mostraram-se eficientes. A SMS-69 (= SEMLA 602-RS), isclada de amendoim, não nodulou nas condiçōes deste ensaio. A observação de outros autores (4) de que ela nodulou o cultivar TMV-8 sugere especificidade nesta espécie de planta, ou uma alteração em relação à cultura da bactéria originalmente introduzida.

O fato de várias estirpes isoladas de leguminosas tropicais não terem nodulado o amendoim já foi observado em ensaios anteriores, indicando certa especificidade $(\boldsymbol{2}, \mathbf{8})$. Especificidade em nivel de cultivares, como ocorre em algumas leguminosas tropicais, não foi ainda relatada para o amendoim (6). Existem, porém, indicações de cque alguns deles apresentam maior eficiência de fixação (3).

\section{CONCLUSÕES}

1. Das 35 estirpes de Rhizobium sp. tipo "cow-pea" testadas, apenas doze nodularam o amendoim; destas, sete se mostraram eficientes fixadoras de $\mathrm{N}_{2}$.

2. As estirpes SMS-139 (= CB 756, Austrália), SMS-176 (=- D 17b, Checoslováquia), SMS-319 e SMS-400 (= 411/MAR. Zimbabwe), foram as mais eficientes, sendo recomendadas para outros estudos.

\section{SUMMARY}

\section{PRELIMINARY SELECTION OF PEANUT RHUZOBIUM SP. STRAINS}

An experiment was carried out in Leonard jars, in the greenhouse, with nitrogen-free nutrient solution to test the efficiency of 35 strains of rhizobia isolated from 15 species of tropical legumes. Twelve of the tested strains were capable of noduie formation in peanut. Seven of those strains were isolated from the trible Hedysareae, which includes the genus Arachis. Only four of the rhizobia strains with inducing nodulation were effective. Dry weight and number of nodules were not good criteria for evaluating effectiveness.

\section{REFERENCIAS BIBLIOGRAFICAS}

1. BATAGLIA, O.C.; TEIXETRA, J. P. F; FURLANI, P. R.; FURLANI, A. M. C. Análise química de plantas. Campinas, Instituto Agronômico, 1978. 31p. (Circular, 87) 
2. BURTON, J. C. Host specificity among certain plants in the «Cow-pea» cross-inoculation group. Soil Science Society of American Proceedings, 16:356$358,1952$.

3. - Pragmatic aspects of the Rhizobium: Leguminous plant association. In: Newton, William E. \& Nyman C.J. SIMPOSIUM OF THE NTTROGEN FIXATION, 1. Proceedings. Washington, University Press, 1976. p.429-446.

4. DADARWAL, K.R.; SINGH, C.S.; SUBBA RAO, N.S. Nodulation and serological studies of rhizobia from six species of Arachis. Flant and Soil, 40:535-544, 1974.

5. LOPES, E. S. Coleção de estirpes de Rhizobium spp. do Instituto Agronômico. Campinas, Instituto Agronômico, 1972. 12p. (Circular, 11)

6. - Ecology of legume-Rhizobium symbiosis. In: DöBEREINER, J.; BURRIS, R. H.; HOLLANDER, A., ed. Limitations and Potentials for Biological Nitrogen Fixation in the Tropics. New York, Plenum Press, 1977.

7. - - ; GIARDINI, A. R.; KIIHL, R. A. S.; IGUE, T. Especificidade hospedeira e pré-seleção de estirpes de Rhizobium japonicum para as variedades Santa-Rosa, Viçoja e IAC-2 de soja. Bragantia, Campinas, 35:1-11, 1976.

8. — Seleção de estirpes de Rhizobium spp. para amendoim (Arachis hypogaea L.) e galáxia (Galactia striata (Jacq.) Urban.). Bragantia, Campinas, 33: CV-CX, 1974. (Nota, 21)

9.

-; SAVY FILHO, A.; OLIVEIRA, M. L. C.; GIARDINI, A. R.; POMPEO, A. S. Observação da nodulação natural em cultivares de amendoim. Bragantia, Campinas, 35:XI-XIII, 1974. (Nota, 3)

10. — - TELLA, R.; ROCHA, J. L. V.; IGUE, T. Inoculação de sementes de amendoim (Arachis hypogaea L.). Bragantia, Campinas, 31:XXVII-XXXIV, 1972. (Nota, 6)

11. NORRIS, D. O. Techniques used in work Rhizobium. In: SOME concepts and methods in subtropical pasture research. England, Commonwealth Agricultural Bureaux, 1964. p.186-198.

12. PROGNôstico. São Paulo, Instituto de Economia Agrícola, 1975/76. 226p.

13. SAVY FILHO, A.; ROSTON, J. A.; LASCA, D. H. de C.; NAKAGAWA, J.; TRANI, P. E.; BELLINAZZI JR., R.; DIEHL, S. R. L. Adubação e calagem para a cultura do amendoim. Campinas, Coordenadoria de Assistência Técnica Integral, 1977. 5p. (Mimeografado) 